\title{
The Correlation between sST2 and Atrial Fibrillation and Its Clinical Significance
}

\author{
Ling Kong, Pei Hu, Chengbin Li, Tao Jiang, Gang Hu \\ School of Medical, Yangtze University, Jingzhou, China \\ Email:775134199@qq.com
}

How to cite this paper: Kong, $\mathrm{L}$., $\mathrm{Hu}, \mathrm{P}$., Li, C.B., Jiang, T. and Hu, G. (2020) The Correlation between sST2 and Atrial Fibrillation and Its Clinical Significance. Yangtze Medicine, 4, 277-283.

https://doi.org/10.4236/ym.2020.44025

Received: December 6, 2019

Accepted: September 27, 2020

Published: September 30, 2020

Copyright $\odot 2020$ by author(s) and Scientific Research Publishing Inc. This work is licensed under the Creative Commons Attribution International License (CC BY 4.0).

http://creativecommons.org/licenses/by/4.0/

cc) (i) Open Access

\begin{abstract}
Objective: To explore the level of soluble growth-stimulated expression gene 2 (sST2) in patients with atrial fibrillation (AF) and its relationship with atrial remodeling. Methods: From December 2018 to July 2019, 86 patients were enrolled in the cardiovascular department of Jingzhou Central Hospital in Hubei Province. AF group was 60 patients with AF diagnosed according to the diagnostic criteria of electrophysiology and pacing branch of Chinese Medical Association, while the control group was 26 normal ECG patients with sinus rhythm examined by ECG and/or DCG. Clinical data, NT proBNP and birth rate of all patients were collected chemical indicators. All the patients in the groups were examined by ECG and color Doppler echocardiography. The serum level of ST2 was detected by enzyme-linked immunosorbent assay (ELISA), and the indexes related to atrial remodeling in echocardiography, left atrial diameter (LAD), were collected, compared and analyzed. Results: Serum sST2 levels and LAD and RAD values in the AF group were higher than those in the control group $(\mathrm{P}<0.05$ or $\mathrm{P}<0.01)$. Serum sST2 levels were higher in the $\mathrm{AF}$ group than in the paroxysmal atrial fibrillation group $(\mathrm{P}=0.031) .1$ ) The sST2 level was positively correlated with LAD and RAD measured by cardiac ultrasound $(r=0.388, P=0.029)$. Conclusion: Serum sST2 levels have early recognition value in patients with high-risk atrial fibrillation, which contributes to the stratification of risk of atrial fibrillation and the prediction of disease activity. Elevated serum sST2 levels may be associated with atrial remodeling. sST2 may be an objective biomarker that predicts the risk of emergency admission in patients with atrial fibrillation. Elevated sST2 levels may be associated with atrial fibrillation progression.
\end{abstract}

\section{Keywords}

Atrial Fibrillation, Atrial Remodeling, Soluble Growth Stimulator Gene 2 


\section{Introduction}

At present, the mechanism of atrial fibrillation (AF) has been studied extensively, but its specific pathological mechanism is still unclear. More and more research results support that atrial remodeling caused by AF plays an important role in AF [1]. Recently, it has been pointed out that soluble growth stimulating gene 2 (soluble growth stimulation expressed gene 2, sST2) may be involved in the development of atrial fibrillation. Therefore, this study aims to explore the level of sST2 in patients with atrial fibrillation and its relationship with atrial remodeling. As one of the members of the interleukin-1 receptor superfamily, ST2 mainly exists in soluble ST2 (sST2) and trans model ST2 (ST2L) [2]. At present, it has been found that high concentration of sST2 can competitively bind to IL-33 as a "decoy receptor". By inhibiting the anti-fibrosis effect of ST2L/IL-33 pathway in the heart, it can promote abnormal cardiac hyperplasia and cardiac remodeling [3] [4] [5]. As a biomarker of myocardial fibrosis, sST2 may also be related to AF [6]. Therefore, high concentration of sST2 in patients with AF plays an important role in the risk stratification and disease activity prediction of AF. At present, an imaging index to evaluate atrial remodeling in clinical work is atrial diameter. Therefore, according to the adult echocardiography standard recommended by ASE/EACVI [7] in 2015, this study studies the progress of sST2 and atrial fibrillation and its relationship with atrial remodeling by comparing the correlation between serum sST2 and atrial diameter.

\section{Data and Methods}

\subsection{Subjects}

The AF group randomly selected 60 patients who were admitted to the cardiovascular department of Jingzhou Central Hospital of Hubei Province from December 2018 to July 2019, including 34 males and 26 females, aged (65.44 \pm 7.73$)$ years. The diagnosis and classification criteria of AF Group [8]: the criteria formulated by the electrophysiology and pacing branch of the Chinese Medical Association: subjective symptoms of palpitation and chest distress and evidence of abnormal ECG and/or DCG: P wave disappeared during AF, F wave replaced, frequency 350 - 600 times/min, the QRS rhythm is absolutely irregular, which shows that the RR interval is uneven, and the QRS wave shape is mostly normal. If it is accompanied by indoor differential conduction or by-pass, it can cause QRS wave width deformity. Exclusion criteria: Patients with acute and chronic bacterial or viral infection, active asthma, chronic obstructive pulmonary disease, acute pulmonary embolism, pregnancy, malignant tumor, tuberculosis, rheumatism and immune system disease, chronic liver and kidney dysfunction, congenital heart disease, cardiomyopathy, valve disease and heart failure. In the control group, 26 normal ECG patients without the diseases listed in the above exclusion criteria were selected, including 13 males and 7 females, aged (60.12 \pm 8.02) years. The clinical data and biochemical indexes of all patients were collected. This study was approved by the clinical research ethics committee of the 
Second Clinical Medical College of Yangtze University. All the subjects signed informed consent forms.

\subsection{Research Methods}

All the selected subjects collected about $5 \mathrm{ml}$ of venous blood in the morning of the second day after admission and placed it in the coagulating blood vessels. They were centrifuged at $4^{\circ} \mathrm{C}$ at $3000 \mathrm{r} / \mathrm{min}$ for $10 \mathrm{~min}$, sealed and sealed after serum separation, and stored in a low-temperature refrigerator at $-70^{\circ} \mathrm{C}$ for testing. After the centralized collection of blood samples, the serum level of sST2 was detected with the sST2 detection kit (enzyme-linked immunosorbent assay, American C \& D Company), and the operation steps were strictly in accordance with the instructions of the kit. At the same time, venous blood was collected and sent to the hospital laboratory to be tested by the professional physician of the laboratory of the hospital for blood routine test, blood biochemistry and other indicators. The left atrium diameter (Left atrial diameter, LAD) and right atrium diameter (Right atrial diameter, RAD) were measured by the specialists in the ultrasound room of our hospital.

\subsection{Statistical Analysis}

$\mathrm{S}-\mathrm{W}$ method is used to test the normality of measurement data. The measurement data conforming to the normal distribution is expressed in $x \pm s$, and the analysis of variance is used for the comparison between groups; the measurement data not conforming to the normal distribution is expressed in $M(Q L$, $\mathrm{QR}$ ), and the comparison between groups is expressed in Kruskal-Wails $\mathrm{H}$ test. Spearman correlation analysis was used to test the correlation of measurement data. Taking the grouping of AF as the dependent variable, the items with statistical significance in single factor analysis as the independent variable, and at the level of $\alpha=0.10$, the logistic regression analysis of multi-classification disorder was carried out. The difference was statistically significant $(\mathrm{P}<0.05)$.

\section{Results}

\subsection{Comparison of General Clinical Data of Subjects}

There was no significant difference in age, gender, height, weight, diabetes mellitus, coronary heart disease, smoking history and drinking history between paroxysmal AF group and persistent $\mathrm{AF}$ group and control group.

\subsection{Comparison of Serum NT-ProBNP, LAD and Serum sST2 Levels}

The level of NT proBNP in paroxysmal atrial fibrillation group and persistent atrial fibrillation group was significantly higher than that in the control group ( $\mathrm{P}$ $<0.05)$. Lad in paroxysmal AF group and persistent AF group was significantly higher than that in control group $(\mathrm{P}<0.05)$. The level of serum ST2 in persistent atrial fibrillation group was higher than that in paroxysmal atrial fibrillation 
group and control group $(\mathrm{P}<0.05)$ (see Table 1$)$.

\subsection{Correlation between NT-ProBNP, LAD and SST2 in AF Group}

There was a positive correlation between NT proBNP and ST2 $(r=0.314, \mathrm{P}<$ 0.05). It is suggested that both of them have the same function in AF, suggesting that $\mathrm{AF}$ is not good after rain (see Table 2). LAD was positively correlated with serum ST2 $(r=0.523, P<0.05)$. It is suggested that both of them have the same function in $\mathrm{AF}$, suggesting that $\mathrm{AF}$ is not good after rain (see Table 2).

\subsection{Serum sST2 Was Associated with Persistent AF ( $<$ < 0.05)}

The risk of sustained AF increased by 1.447 times for every unit increase of serum sST2. There was no significant correlation between serum SST2 and paroxysmal AF ( $P$ > 0.05), but the risk of paroxysmal AF increased by 0.950 times for each additional unit of serum sST2, but it remains to be studied (see Table 3).

Table 1. Comparison of the results of NT-proBNP, LAD and sST2 levels in each group $[\mathrm{M},(\mathrm{QL}, \mathrm{QR}]$.

\begin{tabular}{|c|c|c|c|c|c|c|c|c|c|c|}
\hline \multirow{2}{*}{ Grouping } & \multirow{2}{*}{$\begin{array}{l}\text { Number } \\
\text { of cases }\end{array}$} & \multicolumn{3}{|c|}{ NT-ProBNP (ng/ml) } & \multicolumn{3}{|c|}{$\mathrm{LAD}(\mathrm{cm})$} & \multicolumn{3}{|c|}{$\mathrm{sST} 2(\mathrm{pg} / \mathrm{ml})$} \\
\hline & & $\mathbf{M}$ & P25 & P75 & $\mathbf{M}$ & P25 & P75 & $\mathbf{M}$ & P25 & P75 \\
\hline $\begin{array}{l}\text { Paroxysmal atrial } \\
\text { fibrillation }\end{array}$ & 35 & 465.0 & 200.0 & 900.0 & 3.9 & 3.7 & 4.6 & 17.7 & 11.2 & 24.4 \\
\hline $\begin{array}{l}\text { Persistent atrial } \\
\text { fibrillation }\end{array}$ & 25 & $1120.0^{\circ}$ & 715.5 & 1693.5 & 4.5 & 4.0 & 5.7 & $39.9^{\circ}$ & 37.2 & 49.3 \\
\hline Control group & 25 & $50.0^{* \Delta}$ & 36.5 & 68.0 & $3.3^{* \Delta}$ & 3.2 & 3.6 & $14.3^{\triangle}$ & 10.3 & 19.5 \\
\hline
\end{tabular}

Note: "*" indicates that there is significant difference between the control group and the paroxysmal AF group; " $\Delta$ " indicates that there is a significant difference between the control group and the persistent AF group; " " indicates that there is significant difference between paroxysmal AF group and persistent AF group. $\mathrm{P}<0.05$ was statistically significant.

Table 2. Correlation analysis of serum ST2 level with NT proBNP and LAD in AF group.

\begin{tabular}{ccc}
\hline \multirow{2}{*}{ Research factors } & \multicolumn{2}{c}{ Serum ST2 level $(\mathrm{pg} / \mathrm{ml})$} \\
\cline { 2 - 3 } & r value & P value \\
\hline NT-ProBNP $(\mathrm{ng} / \mathrm{ml})$ & 0.523 & $<0.05$ \\
LAD $(\mathrm{cm})$ & 0.314 & $<0.05$
\end{tabular}

Table 3. Regression analysis of NT-proBNP, LAD and sST2 in serum of AF patients.

\begin{tabular}{cccccccc}
\hline \multicolumn{2}{c}{ Project } & $\begin{array}{c}\text { Regression } \\
\text { coefficient }\end{array}$ & $\begin{array}{c}\text { Standard error } \\
\text { of regression } \\
\text { coefficient }\end{array}$ & wald & P & OR & 95\% CI \\
\hline $\begin{array}{c}\text { Paroxysmal } \\
\text { atrial }\end{array}$ & NT-proBNP & 0.016 & 0.007 & 5.503 & 0.019 & 1.016 & $1.003-1.030$ \\
fibrillation & SST2 & -0.052 & 0.066 & 0.618 & 0.432 & 0.95 & $0.835-1.080$ \\
$\begin{array}{c}\text { Persistent } \\
\text { atrial }\end{array}$ & NT-proBNP & 0.014 & 0.007 & 4.292 & 0.038 & 1.014 & $1.001-1.028$ \\
fibrillation & LAD & 3.472 & 1.98 & 3.075 & 0.08 & 32.203 & $0.664-1560.776$ \\
\hline
\end{tabular}




\section{Discussion}

At present, many studies have determined the predictive effect of serum sST2 level on myocardial infarction and heart failure [9] [10] [11]. In 2018, a study found that the serum concentration of sST2 in patients with AF was significantly higher than that in the healthy control group, and the serum concentration of sST2 in patients with persistent AF was higher than that in patients with paroxysmal AF in the AF subgroup, suggesting that the increased serum concentration of sST2 may play an important pathophysiological role in the development of AF [12]. The results of this study also showed that the correlation between AF and serum sST2 level was higher than that of the control group. Other studies have shown that BNP level in persistent $\mathrm{AF}$ is higher than that in paroxy-small AF [13]. BNP level in patients with paroxysmal AF was significantly higher than that in patients with sinus rhythm [14]. The findings of this study are consistent with this. More and more studies have found that BNP is useful in predicting some cardiovascular diseases, but it has limitations, such as age or renal function and other factors may affect the results, and sST2 may play a role in supplementing and replacing these limitations of BNP. Another important finding of this study is that there is a positive correlation between sST2 and LAD. Cardiac hemodynamic overload will lead to atrial relaxation, and atrial dilation is the main sign of left atrial remodeling. Atrial remodeling promotes the occurrence or maintenance of atrial fibrillation. Cardiac hemodynamic overload will lead to atrial relaxation, and atrial dilation is the main sign of left atrial remodeling. Atrial remodeling promotes the occurrence or maintenance of atrial fibrillation [15], and may also stimulate the secretion of sST2 and BNP [16]. The results of this study also showed that the LAD of patients with AF was higher, and there were also statistical differences in the levels of serum sST2, NT-proBNP and LAD among different AF subgroups. These are consistent with the above results. Of course, there are some shortcomings in this study, such as the small number of cases, the lack of detection of serum sST2 level in patients undergoing atrial fibrillation ablation, so the results still need more clinical research to confirm. In conclusion, the increase of serum sST2 level may be involved in the progress of atrial fibrillation and may be related to atrial remodeling, which may be a potential marker of atrial fibrillation. At the same time, the combination of sST2 and NT-proBNP is more helpful to judge the cardiac remodeling after AF. Therefore, serum sST2 can be used as a biomarker for the activity of atrial fibrillation disease, as an independent biomarker for the prediction of other cardiovascular diseases, especially heart failure in patients with atrial fibrillation, and can be used to guide clinical treatment.

\section{Summary}

In conclusion, we found that the increase of serum ST2 concentration may play an important pathophysiological role in the development of atrial fibrillation, and it is related to atrial remodeling, which suggests that the increased serum 
ST2 level can be used as a biomarker of the activity of atrial fibrillation disease, and it can predict the recurrence of persistent atrial fibrillation after the treatment, which is helpful to our atrial fibrillation. The clinical diagnosis and treatment of the disease have important guiding significance.

\section{Acknowledgements}

Thank you for the experimental platform and technical support provided by the Department of cardiovascular medicine and Laboratory of the Second Clinical Medical College of Yangtze University.

\section{Conflicts of Interest}

The authors declare no conflicts of interest regarding the publication of this paper.

\section{References}

[1] Dzeshka, M.S., Lip, G.Y.H., Viktor, S., et al. (2015) Cardiac Fibrosis in Patients with Atrial Fibrillation: Mechanisms and Clinical Implications. Journal of the American College of Cardiology, 66, 943-959. https://doi.org/10.1016/j.jacc.2015.06.1313

[2] Karayannis, G., Triposkiadis, F., Skoularigis, J., et al. (2013) The Emerging Role of Galectin-3 and ST2 in Heart Failure: Practical Considerations and Pitfalls Using Novel Biomarkers. Current Heart Failure Reports, 10, 441-449. https://doi.org/10.1007/s11897-013-0169-1

[3] Sanada, S., Hakuno, D., Higgins, L.J., et al. (2007) IL-33 and ST2 Comprise a Critical Biomechanically Induced and Cardioprotective Signaling System. The Journal of Clinical Investigation, 117, 538-549. https://doi.org/10.1172/JCI30634

[4] Kim, M.S., Jeong, T.D., Han, S.B., et al. (2015) Role of Soluble ST2 as a Prognostic Marker in Patients with Acute Heart Failure and Renal Insufficiency. Journal of Korean Medical Science, 30, 569-575. https://doi.org/10.3346/jkms.2015.30.5.569

[5] Shah, R.V. and Januzzi, J.L. (2014) Soluble ST2 and Galectin-3 in Heart Failure. Clinics in Laboratory Medicine, 34, 87-97. https://doi.org/10.1016/j.cll.2013.11.009

[6] Chen, C.X., Qu, X., Gao, Z., et al. (2018) Soluble ST2 in Patients with Nonvalvular Atrial Fibrillation and Prediction of Heart Failure. International Heart Journal, 59, 58-63. https://doi.org/10.1536/ihj.16-520

[7] Lang, R.M., Badano, L.P., et al. (2015) Recommendations for Cardiac Chamber Quantification by Echocardiography in Adults: An Update from the American Society of Echocardiography and the European Association of Cardiovascular Imaging. Journal of the American Society of Echocardiography, 28, 1-39.e14. https://doi.org/10.1016/j.echo.2014.10.003

[8] Electrophysiology and Pacing Branch of Chinese Medical Association, Professional Committee of Cardiology of Chinese Medical Association (2018) Atrial Fibrillation: Current Understanding and Treatment Suggestions 2018. Chinese Journal of Cardiac Pacing and Electrophysiology, 32, 315-368.

[9] Xu, S.D., Su, G.H., Lu, Y.X., et al. (2014) Elevated Soluble ST2 and Depression Increased the Risk of All-Cause Mortality and Hospitalization in Patients with Heart Failure. International Heart Journal, 55, 445-450. https://doi.org/10.1536/ihj.13-371

[10] Minamisawa, M., Motoki, H., Izawa, A., et al. (2016) Comparison of Inflammatory 
Biomarkers in Outpatients with Prior Myocardial Infarction. International Heart Journal, 57, 11-17. https://doi.org/10.1536/ihj.15-197

[11] Wang, T.J., Wollert, K.C., Larson, M.G., et al. (2012) Prognostic Utility of Novel Biomarkers of Cardiovascular Stress: The Framingham Heart Study. Circulation, 126, 1596-1604. https://doi.org/10.1161/CIRCULATIONAHA.112.129437

[12] Ma, X., Yuan, H., Luan, H.-X., et al. (2018) Elevated Soluble ST2 Concentration May Involve in the Progression of Atrial Fibrillation. Clinica Chimica Acta, 480, 138-142. https://doi.org/10.1016/j.cca.2018.02.005

[13] Yamada, T., Murakami, Y., Okada, T., et al. (2007) Plasma Brain Natriuretic Peptide Level after Radiofrequency Catheter Ablation of Paroxysmal, Persistent, and Permanent Atrial Fibrillation. Europace, 9, 770-774. https://doi.org/10.1093/europace/eum157

[14] Seegers, J., Zabel, M., Grüter, T., et al. (2015) Natriuretic Peptides for the Detection of Paroxysmal Atrial Fibrillation. Open Heart, 2, e000182. https://doi.org/10.1136/openhrt-2014-000182

[15] Nattel, S., Burstein, B. and Dobrev, D. (2008) Atrial Remodeling and Atrial Fibrillation: Mechanisms and Implications. Circulation: Arrhythmia and Electrophysiolo$g y, 1,62-73$. https://doi.org/10.1161/CIRCEP.107.754564

[16] Chen, C.X., Qu, X., Gao, Z., et al. (2018) Soluble ST2 in Patients Nonvalvular Atrial Fibrillation and Prediction of Heart Failure. International Heart Journal, 59, 58-63. https://doi.org/10.1536/ihj.16-520 